\title{
ASYMPTOTIC PROPERTIES OF THE SOLUTIONS OF A CLASS OF OPERATOR-DIFFERENTIAL EQUATIONS
}

\author{
A. AKÇA \\ Akdeniz University \\ Department of Mathematics \\ Antalya, TURKEY \\ D.D. BAINOV \\ South-Western University \\ Department of Mathematics \\ Blagoevgrad, BULGARIA \\ M.B. DIMITROVA \\ Technical University \\ Department of Mathematics \\ Sliven, BULGARIA
}

(Received March, 1993; revised April, 1994)

\begin{abstract}
Some asymptotic properties of the nonoscillating solutions of operatordifferential equations of arbitrary order are investigated.
\end{abstract}

Key words: Asymptotic Properties, Operator Differential Equations.

AMS (MOS) subject classifications: 34K35.

\section{Introduction}

The goal of the present paper is, by means of a single approach, to investigate some asymptotic properties of the nonoscillating solutions of differential equations with "maximum", with distributed delay, with autoregulable deviation, with integro-differential equations, and with other properties. To realize this single approach, an operator with certain properties is introduced, as well as appropriately chosen operator-differential equations and inequalities. In the paper, some results obtained in [2]-[4], [6], [7], [10], [11] are generalized.

Each of the concrete realizations of the operator introduced generates a class of differential equations which find application in the theory of optimal control, theoretical physics, population dynamics, pharmacokinetics and economics.

We shall note that the ordinary differential equations with "maxima" (where the operator $\mathcal{A}$ considered has the form $\left.(\mathcal{A} x)(t)=\max _{s \in[g(t), t]}(x)\right)$ are still seldom studied. On the other hand, such equations arise, for instance, in the mathematical simulation of some systems with automatic regulation. We shall illustrate the above by an example - a mathematical model of a system for automatic regulation of the voltage of a constant current generator ([5], [8]). 
The object regulated is a constant current generator with parallel stimulation. The quantity regulated is the voltage between the clamps of the generator which feeds an electric circuit with various consumers. External disturbances $f(t)$ occur under a change of the consumption of electricity in the circuit or under a change of the normal angular velocity of the rotor of the generator. They cause an undesirable deviation of the voltage $u(t)$ from the previously fixed regime $u_{0}=$ const. The automatic regulator is intended to maintain a constant voltage $u_{0}$ under external disturbances. It consists of a measuring device and of a regulating device which are connected in series. Under a deviation $x(t)$ of the voltage from the fixed stationary regime $u_{0}$, the regulating device (rheostat) changes the resistance $\tau$ of the stimulating circuit. Then as a result of this correction $\delta=\Delta \tau$, the voltage produced returns to the stationary regime $u_{0}$.

The dynamics of the process of regulation of the voltage is described by the equation

$$
T_{0} x^{\prime}(t)+x(t)=-\kappa_{0} \delta(t)+f(t)
$$

where $x(t)$ is the deviation of the voltage from the given regime $u_{0}, T_{0}$ is a constant of time dimension which depends on the inductivity of the stimulating circuit and on the static characteristics of the generator, $\kappa_{0}$ is the amplification factor of the object, and $f(t)$ the external effect.

On the other hand, the equation of the "ideal" regulator which corresponds to the maximal deviation of the voltage in an interval of time $h$ has the form

$$
\delta(t)=\kappa_{1} \cdot \max \{x(s), s \in[t-h, t]\}
$$

where $\kappa_{1}$ is the amplification factor of the regulator. Then the equation of the system for automatic regulation of the voltage of a constant current generator takes on the form

$$
T_{0} x^{\prime}(t)+x(t)+\kappa_{0} \kappa_{1} \cdot \max \{x(s), s \in[t-h, t]\}=f(t) .
$$

\section{Preliminary Notes}

Consider the operator-differential equation

$$
\left[\tau_{n-1}(t)\left[\tau_{n-2}(t)\left[\ldots\left[\tau_{0}(t) x(t)\right]^{\prime} \ldots\right]^{\prime}\right]^{\prime}\right]^{\prime}+\delta F(t,(\mathcal{A} x)(t))=b(t)
$$

for $t \geq t_{0}$, where $t_{0} \in \mathbb{R}$ is a fixed number, $n \geq 1$ is an integer, $\mathcal{A}$ is an operator with certain properties, $\delta= \pm 1$ and

$$
\tau_{i} \in C^{n-i}\left(\left[t_{0}, \infty\right) ;(0, \infty)\right), \quad i=0,1, \ldots, n-1, \quad b \in C\left(\left[t_{0}, \infty\right) ; \mathbb{R}\right) .
$$

Introduce the following notation:

$$
\begin{gathered}
\left(L_{0} x\right)(t)=\tau_{0}(t) x(t) \\
\left(L_{i} x\right)(t)=\tau_{i}(t)\left[\left(L_{i-1} x\right)(t)\right]^{\prime}, 1 \leq i \leq n, \tau_{n}(t) \equiv 1,
\end{gathered}
$$

where $x:\left[T_{x}, \infty\right) \rightarrow \mathbb{R}, T_{x} \geq t_{0}$.

Denote by $\mathscr{D}_{n}$ the set of all functions $x \in C\left(\left[T_{x}, \infty\right), \mathbb{R}\right)$ such that the functions $L_{i} x$ $(0 \leq i \leq n)$ exist and are continuous in $\left[T_{x}, \infty\right)$. 
Definition 1: The function $x$ is said to be a solution of equation (1) if $x \in \mathscr{D}_{n}$ and $x$ satisfies equation (1) for $t \geq \max \left\{T_{x}, T_{A x}\right\}$.

Definition 2: A given function $u:\left[t_{0}, \infty\right) \rightarrow \mathbb{R}$ is said to eventually enjoy the property $P$ if there exists a point $t_{p, u} \geq t_{0}$ such that for $t \geq t_{p, u}$ it enjoys the property $P$.(See note from proofer)

Definition 3: The solution $x$ of equation (1) is said to be regular if $\sup \{|x(t)|\}>0$ eventually.

Definition 4: The regular solution $x$ of equation (1) is said to oscillate if $\sup \{t, x(t)=0\}=$ $\infty$. Otherwise, the regular solution $x$ is said to be nonoscillating.

Introduce the following conditions:

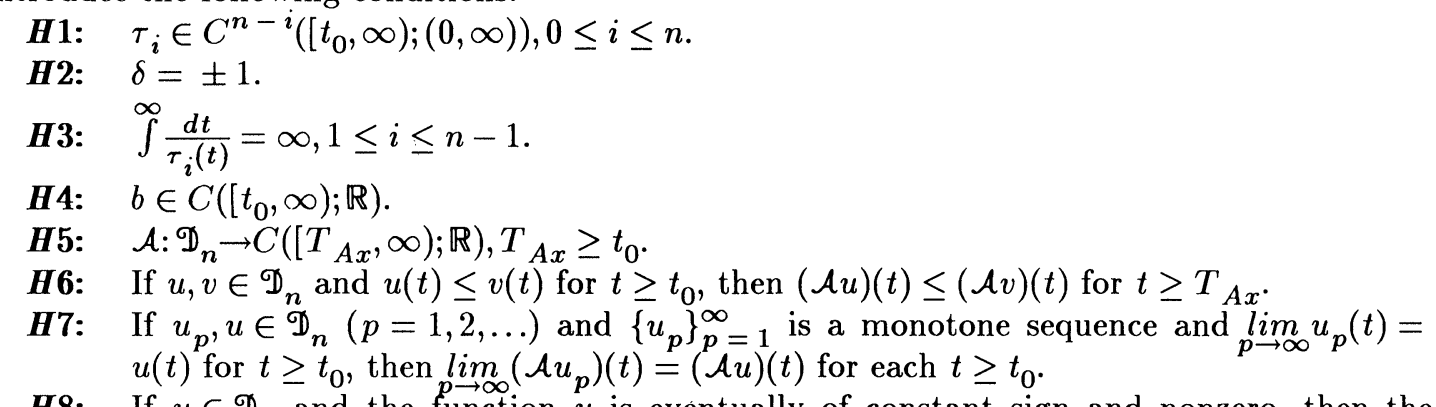

H8: If $u \in \mathscr{D}_{n}$ and the function $u$ is eventually of constant sign and nonzero, then the function $\mathcal{A} u$ is also eventually of constant sign and nonzero, and they have the same sign.

H9: $\quad F \in C\left(\left[t_{0}, \infty\right) \times\left(\mathbb{R}_{+} \cup \mathbb{R}_{-}\right)\right), \mathbb{R}_{+}=(0, \infty), \mathbb{R}_{-}=(-\infty, 0)$.

Lemma 1 [11]: Let the following conditions hold:

1. Conditions $\mathrm{H} 1-\mathrm{H} 3$ are met.

2. $x \in \mathscr{D}_{n}, x(t)>0$ for $t \geq T\left(T \geq t_{0}\right)$.

3. $\quad\left(L_{n} x\right)(t)$ is of constant sign in $[T, \infty)$.

Then there exists an integer $\ell$ such that:

1. For $\left(L_{n} x\right)(t) \leq 0, n+\ell$ is an odd number.

2. For $\left(L_{n} x\right)(t) \geq 0, n+\ell$ is an even number.

3. $(-1)^{\ell+} j\left(L_{j} x\right)(t) \geq 0$ for $\ell \leq n-1, j=\ell, \ldots, n-1 ; t \geq T$.

4. $\left(L_{i} x\right)(t)>0$ for $\ell>1,1 \leq i \leq \ell-1, t \geq T$.

Lemma 2 [11]: Let the following conditions hold:

1. Condition $H 1$ is satisfied.

2. $0<\lim _{t \rightarrow \infty} \inf \tau_{i}(t) \leq \lim _{t \rightarrow \infty} \sup \tau_{i}(t)<\infty, 1 \leq i \leq n-1$.

3. $x \in \mathscr{D}_{n}$.

Then, if one of the following two conditions hold:

1. $L_{0} x$ is a bounded function in $[T, \infty)$ and $\lim _{t \rightarrow \infty}\left(L_{n} x\right)(t)=0$.

2. $L_{n} x$ is a bounded function in $[T, \infty)$ and $\lim _{t \rightarrow \infty}\left(L_{0}, x\right)(t) \in \mathbb{R}$, then $\lim _{t \rightarrow \infty}\left(L_{i} x\right)(t)=0$, $1 \leq i \leq n-1$.

For any function $y \in C([T, \infty) ; \mathbb{R})$ and for any integer $\ell, 0 \leq \ell \leq n$ define the function 


$$
\begin{aligned}
& \left(\frac{1}{\tau_{0}(t)} \int_{t}^{\infty} \frac{1}{\tau_{1}\left(s_{1}\right)} \int_{s_{1}}^{\infty} \frac{1}{\tau_{2}\left(s_{2}\right)} \ldots \int_{s_{n-2}}^{\infty} \frac{1}{\tau_{n-1}\left(s_{n-1}\right)} \int_{s_{n-1}}^{\infty} y(s) d s d s_{n-1} \ldots d s_{2} d s_{1}\right. \\
& \text { for } \ell=0 \\
& \left(\varphi_{\tau}^{\ell} y\right)(t)=\left\{\frac{1}{\tau_{0}(t)} \int_{T}^{t} \frac{1}{\tau_{1}\left(s_{1}\right)} \int_{T}^{s_{1}} \frac{1}{\tau_{2}\left(s_{2}\right)} \ldots \int_{T}^{s_{\ell}-1} \frac{1}{\tau_{\ell}\left(s_{\ell}\right)} \int_{s_{\ell}}^{\infty} \frac{1}{\tau_{\ell+1}\left(s_{\ell+1}\right)} \ldots \int_{s_{n-1}}^{\infty} y(s) d s \ldots d s_{\ell} d s_{\ell-1} \ldots d s_{2} d s_{1}\right. \\
& \text { for } 0<\ell \leq n-1 \\
& \frac{1}{\tau_{0}(t)} \int_{T}^{t} \frac{1}{\tau_{1}\left(s_{1}\right)} \int_{T}^{s_{1}} \frac{1}{\tau_{2}\left(s_{2}\right)} \ldots \int_{T}^{s_{n-2}} \frac{1}{\tau_{n-1}\left(s_{n-1}\right)} \int_{T}^{s_{n-1}} y(s) d s d s_{n-1} \ldots d s_{2} d s_{1} \text { for } \ell=n
\end{aligned}
$$

\section{Main Results}

Theorem 1: Let the following conditions hold:

1. Conditions H1-H9 are met.

2. There exists a function $w$ defined in $\left[t_{0}, \infty\right)$ such that $w \in \mathscr{D}_{n}$ and $\left(L_{n} w\right)(t)=b(t)$.

3. The function $L_{0} w$ is bounded below in the interval $\left[t_{0}, \infty\right)$.

4. There exists a positive solution $y$ of the inequality

$$
\delta\left(L_{n} x\right)(t)+F(t,(\mathcal{A} x)(t)) \leq \delta \cdot b(t)
$$

such that $\lim _{t \rightarrow \infty} \inf \left(L_{0} y\right)(t)>0$.

5. $\quad F(t, u)>0$ for $(t, u) \in\left[t_{0}, \infty\right) \times \mathbb{R}_{+}$and $F(t, u)$ is an increasing function with respect to $u \in \mathbb{R}_{+}$.

Then there exists a positive solution $x$ of equation (1) with the following properties:

1. $\lim _{t \rightarrow \infty} \inf \left(L_{0} x\right)(t)>0$

2. $x(t) \leq y(t)$ eventually.

Proof: Let $y(t)>0$ be a solution of inequality (2) in the interval $\left[T_{0}, \infty\right)\left(T_{0} \geq t_{0}\right)$ and $\lim _{t \rightarrow \infty} \inf \left(L_{0} y\right)(t)>0$. Then $(\mathcal{A} y)(t)>0$ eventually.

Introduce the following notation:

$$
\begin{gathered}
w_{0}(t)=w(t)-\frac{1}{\tau_{0}(t)} \lim _{t \rightarrow \infty} \inf \left(L_{0} w\right)(t) \\
u(t)=y(t)-w_{0}(t) .
\end{gathered}
$$

Then $0<F(t,(\mathcal{A} y)(t)) \leq-\delta\left(L_{n} u\right)(t)$ eventually, i.e. the function $\left(L_{n} u\right)(t)$ is of constant sign for $t \geq T_{0}$. Hence the function $L_{0} u$ is monotone in $\left[T_{0}, \infty\right)$. This fact implies the existence of $\lim _{t \rightarrow \infty}\left(\bar{L}_{0} u\right)(t) \in \mathbb{R} \cup\{-\infty,+\infty\}$. 
But $\lim _{t \rightarrow \infty}\left(L_{0} u\right)(t)=\lim _{t \rightarrow \infty} \inf \left(L_{0} y\right)(t)>0$. Thus we obtained that $u$ is an eventually positive function. Let $[\tau, \infty), \tau \geq T_{0}$ be the largest interval in which the function $u$ is positive.

From Lemma 1 it follows that there exists an integer $\ell(0 \leq \ell \leq n)$ such that

1. $n+\ell$ is an odd number for $\delta=1$.

2. $n+\ell$ is an even number for $\delta=-1$.

3. $\quad(-1)^{\ell+j}\left(L_{j} u\right)(t) \geq 0$ for $\ell \leq n-1 ; j=\ell, \ldots, n-1 ; t \geq \tau$.

4. $\quad\left(L_{i} u\right)(t)>0$ for $\ell>1,1 \leq i \leq \ell-1, t \geq \tau^{*} \geq \tau$.

Introduce the following notation:

$$
\begin{aligned}
& T=\left\{\begin{array}{ccc}
\tau & \text { for } \ell=0 & \text { or } \ell=1 \\
\tau^{*} & \text { for } \ell>1
\end{array}\right. \\
& \kappa=\left\{\begin{array}{cc}
\lim _{t \rightarrow \infty}\left(L_{0} u\right)(t), & \ell=0 \\
\left(L_{0} u\right)(T), & \ell>0 .
\end{array}\right.
\end{aligned}
$$

From condition $H 1$ and the fact that the function $u$ is eventually positive it follows that $\kappa>0$. From (2) we obtain that $-\kappa<\left(L_{0} w_{0}\right)(t)$ eventually, i.e. $\frac{\kappa}{\tau_{0}(t)}+w_{0}(t)>0$ for $t \geq T$.

After a repeated integration of inequality (2) we obtain that

$$
y(t) \geq \frac{\kappa}{\tau_{0}(t)}+w_{0}(t)+\left(\varphi_{\tau}^{\ell}(F(\cdot, \mathcal{A} y))(t)\right.
$$

Let $X$ be the set of all continuous functions $x$ for $t \geq T$ such that

$$
\frac{\kappa}{\tau_{0}(t)}+w_{0}(t) \leq x(t) \leq y(t)
$$

For any function $x \in X$ define the function $\bar{x}(t)$ :

$$
\bar{x}(t)=\left\{\begin{array}{cc}
x(t), & t \geq T \\
\frac{x(T)}{y(T)} y(t), & T_{0} \leq t \leq T .
\end{array}\right.
$$

From the definition of $\bar{x}(t)$ it follows that

$$
\frac{\kappa}{\tau_{0}(t)}+w_{0}(t) \leq \bar{x}(t) \leq y(t), \quad t \geq T_{0} .
$$

Define the operator $S: X \rightarrow E$ by the formula

$$
(S x)(t)=\frac{\kappa}{\tau_{0}(t)}+w_{0}(t)+\left(\varphi_{\tau}^{\ell}(F(\cdot, \mathcal{A} \bar{x}))\right)(t)
$$

where $E$ is the set of all continuous functions in $\left[T_{0}, \infty\right)$.

The inclusion $S X \subset X$ is valid since:

1. From the definition of the operator $S$ it follows that

$$
\frac{\kappa}{\tau_{0}(t)}+w_{0}(t) \leq(S x)(t), \quad t \geq T .
$$


2. From condition 3 of Theorem 1 and condition $H 6$ we obtain that

$$
y(t) \geq(S x)(t)
$$

Let $x_{1}, x_{2} \in X$ and $0<x_{1}(t) \leq x_{2}(t)$. From the definition of the operator $S$ it follows that $0<\left(S x_{1}\right)(t) \leq\left(S x_{2}\right)(t)$ for $t \geq T_{0}$, i.e. $S$ is a monotone increasing mapping of the set $X$ into itself. Let $\left\{\left.x_{n}(t)\right|_{n=0} ^{\infty}\right.$ be a monotone decreasing sequence of elements of the set $X$ for $t \geq T$ obtained by the following recurrent formula:

$$
\begin{gathered}
x_{0}(t)=y(t), t \geq T \\
x_{n}(t)=\left(S x_{n-1}\right)(t), \quad t \geq T .
\end{gathered}
$$

Let $\lim _{n \rightarrow \infty} x_{n}(t)=x(t)$ for $t \geq T$. Then $\lim _{n \rightarrow \infty}\left(\mathcal{A} x_{n}\right)(t)=(\mathcal{A} x)(t)$. From the Lebesgue dominated convergence theorem we obtain that $\lim _{n \rightarrow \infty}\left(S x_{n}\right)(t)=(S x)(t)$ for $t \geq T$. But from (4) it follows that $\lim _{n \rightarrow \infty}\left(S x_{n}\right)(t)=x(t)$. Then we obtain that $(S x)(t)=x(t)$, i.e. $x(t)$ is the positive solution sought of equation (1) such that $\lim _{t \rightarrow \infty} \inf \left(L_{0} x\right)(t)>0, x(t) \leq y(t)$ eventually.

Theorem 2: Let the following conditions hold:

1. Conditions 1 and 2 of Theorem 1 are met.

2. The function $L_{0} w$ is bounded above for $t \geq t_{0}$.

3. There exists a negative solution $y$ of the inequality

$$
\delta\left(L_{n} x\right)(t)+F(t,(\mathcal{A} x)(t)) \geq \delta b(t)
$$

such that $\lim _{t \rightarrow \infty} \sup \left(L_{0} y\right)(t)<0$.

4. $F(t, u)<0,(t, u) \in\left(\left[t_{0}, \infty\right) \times \mathbb{R}_{-}\right), F(t, u)$ is an increasing function with respect to $u \in \mathbb{R}_{-}$.

Then there exists a negative solution $x$ of equation (1) with the properties:

1. $\lim _{t \rightarrow \infty} \sup \left(L_{0} x\right)(t)<0$

2. $x(t) \geq y(t)$ eventually.

The proof of Theorem 2 is carried out along the scheme of the proof of Theorem 1.

Theorem 3: Let the following conditions hold:

1. Condition 1 of Theorem 2 is met.

2. $\quad$ There exists $\lim _{t \rightarrow \infty}\left(L_{0} w\right)(t) \in \mathbb{R}$.

3. $F(t, u)>0$ for $(t, u) \in\left[t_{0}, \infty\right) \times \mathbb{R}_{+},\left(F(t, u)<0\right.$ for $\left.(t, u) \in\left[t_{0}, \infty\right) \times \mathbb{R}_{-}\right)$.

Then each positive (negative) solution of equation (1) enjoys the property

$$
\lim _{t \rightarrow \infty}\left(L_{0} x\right)(t) \in \mathbb{R} \cup\{-\infty,+\infty\}
$$

Proof: Let $x$ be a positive solution of equation (1) in the interval $[T, \infty), T \geq t_{0}$.

Introduce the notation:

$$
w_{0}(t)=w(t)-\frac{1}{\tau_{0}(t)} \cdot \lim _{t \rightarrow \infty}\left(L_{0} w\right)(t)
$$




$$
u(t)=x(t)-w_{0}(t), t \geq T .
$$

Then $\left(L_{n} u\right)(t)=-\delta F(t,(\mathcal{A} x)(t))$, i.e. $L_{n} u$ is of constant sign in the interval $[T, \infty)$. This implies that $L_{0} u$ is a monotone function for $t \geq T$, i.e. there exists

$$
\lim _{t \rightarrow \infty}\left(L_{0} u\right)(t) \in \mathbb{R} \cup\{-\infty,+\infty\}
$$

But $\lim _{t \rightarrow \infty}\left(L_{0} u\right)(t)=\lim _{t \rightarrow \infty}\left(L_{0} x\right)(t)$, i.e. there also exists $\lim _{t \rightarrow \infty}\left(L_{0} x\right)(t) \in \mathbb{R} \cup\{-\infty,+\infty\}$.

Theorem 4: Let the following conditions hold:

1. Conditions 1,2 and 5 of Theorem 1 hold.

2. There exists a positive solution $y$ of the equation

$$
\left(L_{n} x\right)(t)+\delta F(t,(\mathcal{A} x)(t))=0
$$

such that $\lim _{t \rightarrow \infty}\left(L_{0} y\right)(t)>0$.

Then there exists a positive solution $x$ of equation (1) with the properties:

1. $\lim _{t \rightarrow \infty}\left(L_{0} x\right)(t)>0$,

2. $x(t) \leq y(t)$ eventually.

Proof: Let $y$ be a positive solution of equation (7) in the interval $\left[T_{0}, \infty\right), T_{0} \geq t_{0}$ and $\lim _{t \rightarrow \infty}\left(L_{0} y\right)(t)>0$.

Introduce the notation:

$$
\begin{gathered}
w_{0}(t)=w(t)-\frac{1}{\tau_{0}(t)} \lim _{t \rightarrow \infty}\left(L_{0} w\right)(t) \\
u(t)=y(t)+w_{0}(t) .
\end{gathered}
$$

Then $\lim _{t \rightarrow \infty}\left(L_{0} u\right)(t)=\lim _{t \rightarrow \infty}\left(L_{0} y\right)(t)>0$.

Choose a constant $C$ such that $0<C<\lim _{t \rightarrow \infty}\left(L_{0} u\right)(t)$. Let us choose $T \geq T_{0}$ so that for $t \geq T$, $\left(L_{0} u\right)(t)>C, \quad\left(L_{0} w_{0}\right)(t) \leq C . \quad$ Then for the function $\bar{u}(t)=u(t)-\frac{C}{\tau_{0}(t)}$ we obtain that $0<\bar{u}(t) \leq y(t), t \geq T$.

Consequently, $\bar{u}(t)>0$ is a solution of the inequality

$$
\delta\left(L_{n} \bar{u}\right)(t)+F(t,(\mathcal{A} \bar{u})(t)) \leq \delta b(t) .
$$

Moreover, $\lim _{t \rightarrow \infty}\left(L_{0} \bar{u}\right)(t)=\lim _{t \rightarrow \infty}\left(L_{0} u\right)(t)>0$. From Theorem 1, it follows that there exists a positive solution $x$ of equation (1) such that $\lim _{t \rightarrow \infty}\left(L_{0} x\right)(t)>0, x(t) \leq \bar{u}(t) \leq y(t)$ eventually.

Theorem 5: Let the following conditions hold:

1. Conditions 1 and 2 of Theorem 3 and condition 4 of Theorem 2 are met.

2. There exists a negative solution $y$ of equation (7) such that $\lim _{t \rightarrow \infty}\left(L_{0} y\right)(t)<0$.

Then there exists a negative solution $x$ of equation (1) with the properties:

1. $\lim _{t \rightarrow \infty}\left(L_{0} x\right)(t)<0$ 
2. $\quad x(t) \geq y(t)$ eventually.

The proof of Theorem 5 is carried out along the scheme of the proof of Theorem 4.

Consider the operator-differential equation

$$
\left[\tau_{n-1}^{*}(t)\left[\tau_{n-2}^{*}(t)\left[\ldots\left[\tau_{0}^{*}(t) x(t)\right]^{\prime} \ldots\right]^{\prime}\right]^{\prime}\right]^{\prime}+\delta F(t,(\mathcal{A} x)(t))=0
$$

where $\tau_{i}^{*} \in C^{n-i}\left(\left[t_{0}, \infty\right) ; \mathbb{R}_{+}\right), 0 \leq i \leq n-1$.

Introduce the following notation:

$$
\begin{gathered}
\left(L_{0}^{*} x\right)(t)=\tau_{0}^{*}(t) x(t) \\
\left(L_{i}^{*} x\right)(t)=\tau_{i}^{*}(t)\left[\left(L_{i-1}^{*} x\right)(t)\right]^{\prime}, i=1,2, \ldots, n ; \tau_{n}^{*}(t) \equiv 1
\end{gathered}
$$

Theorem 6: Let the following conditions hold:

1. $\quad \int \frac{d t}{\tau_{i}^{*}(t)}=\infty, 1 \leq i \leq n-1$.

2. $\tau_{i}^{*}(t) \leq \tau_{i}(t)$ for $t \geq t_{0}, 0 \leq n-1$.

3. Conditions H1-H9 and condition 5 of Theorem 1 are met.

4. There exists a positive solution $y$ of equation (8) such that $\lim _{t \rightarrow \infty}\left(L_{0}^{*} y\right)(t)>0$.

Then there exists a positive solution $x$ of equation (7) with the following properties:

1. $\quad \lim _{t \rightarrow \infty}\left(L_{0} x\right)(t)>0$

2. $x(t) \leq y(t)$ eventually.

Proof: Let $y$ be a solution of equation $(8)$ in $\left[T_{0}, \infty\right)$ for $T_{0} \geq t_{0}$ and $\lim _{t \rightarrow \infty}\left(L_{0}^{*} y\right)(t)>0$. Consequently,

$$
\begin{gathered}
\left(L_{n}^{*} y\right)(t)=-\delta F(t,(\mathcal{A} y)(t)) \text { i.e. }\left(L_{n}^{*} y\right)(t) \geq 0 \text { for } \delta=-1 \text { and } \\
\left(L_{n}^{*} y\right)(t) \leq 0 \text { for } \delta=1, t \geq T_{0}
\end{gathered}
$$

From Lemma 1 it follows that there exists an integer $\ell, 0 \leq \ell \leq n$ such that $n+\ell$ is an odd number for $\delta=1, n+\ell$ is an even number for $\delta=-1$ and

$$
\begin{gathered}
(-1)^{\ell+j}\left(L_{j}^{*} y\right)(t) \geq 0, t \geq T_{0}, \ell \leq n-1, \ell \leq j \leq n-1 \\
\left(L_{i}^{*} y\right)(t)>0, t \geq T_{1}, T_{1} \geq T_{0}, \ell>1,1 \leq i \leq \ell-1 .
\end{gathered}
$$

Introduce the following notation:

$$
\begin{gathered}
T=\left\{\begin{array}{cc}
T_{0}, & \ell=0 \\
T_{1}, & \ell>1
\end{array}\right. \\
\kappa=\left\{\begin{array}{cc}
\lim _{t \rightarrow \infty}\left(L_{0}^{*} y\right)(t), & \text { or } \ell=1 \\
\left(L_{0}^{*} y\right)(T), & \ell>0 .
\end{array}\right.
\end{gathered}
$$


Then for $t \geq T$ we obtain that

$$
y(t) \geq \frac{\kappa}{\tau_{0}^{*}(t)}+\left(\varphi_{\tau^{*}}^{\ell}(F(\cdot, \mathcal{A} y))\right)(t)
$$

But from condition 2 of Theorem 6 it follows that

$$
y(t) \geq \frac{\kappa}{\tau_{0}(t)}+\left(\varphi_{\tau}^{\ell}(F(\cdot, \mathcal{A} y))\right)(t)
$$
define

Consider the set $X$ of all continuous functions $x$ in $[T, \infty)$ such that $\frac{\kappa}{\tau_{0}(t)} \leq x(t) \leq y(t)$ and

$$
\bar{x}(t)=\left\{\begin{array}{cc}
x(t), & t \geq T \\
\frac{x(T)}{y(T)} y(t), & T_{0} \leq t \leq T
\end{array}\right.
$$

for each function $x \in X$.

Define the operator $S: X \rightarrow C\left(\left[t_{0}, \infty\right) ; \mathbb{R}\right)$ by the formula

$$
(S x)(t)=\frac{\kappa}{\tau_{0}(t)}+\left(\varphi_{\tau}^{\ell} F(\cdot, \mathcal{A} \bar{x})\right)(t) .
$$

It is immediately verified that

$$
\frac{\kappa}{\tau_{0}(t)} \leq(S x)(t) \leq y(t), t \geq t_{0} \text {, i.e. } S: X \rightarrow X .
$$

Let $x_{1}, x_{2} \in X$ and $x_{1}(t) \leq x_{2}(t), t \geq T$. Then

$$
\left(S x_{1}\right)(t) \leq\left(S x_{2}\right)(t), t \geq T .
$$

Consider the convergent sequence $\left\{x_{\kappa}(t)\right\}_{\kappa=0}^{\infty}, t \geq T$ such that

$$
\begin{gathered}
x_{0}(t)=y(t) \\
x_{\kappa}(t)=\left(S x_{\kappa-1}\right)(t), \kappa=1,2, \ldots
\end{gathered}
$$

Thus the sequence $\left\{x_{\kappa}(t)\right\}_{\kappa=1}^{\infty}$ is decreasing for $t \geq T$. If $x(t)=\lim _{n \rightarrow \infty} x_{n}(t)$ then from the Lebesgue dominated convergence theorem it follows that $x(t)=(S x)(t)$, i.e. $x$ is a positive solution of equation (7) with the properties (9).

Theorem 7: Let the following conditions hold:

1. Conditions 1, 2 and 3 of Theorem 6 and condition 4 of Theorem 2 are met.

2. There exists a negative solution $y$ of equation (8) with the properties $\lim _{t \rightarrow \infty}\left(L_{0}^{*} y\right)(t)<0$.

Then there exists a negative solution $x$ of equation (7) with the following properties:

1. $\lim _{t \rightarrow \infty}\left(L_{0} x\right)(t)<0$,

2. $x(t) \geq y(t)$ eventually.

The proof of Theorem 7 is carried out along the scheme of the proof of Theorem 6 . 
Theorem 8: Let the following conditions hold:

1. Conditions $\mathrm{H1}, \mathrm{H} 2, \mathrm{H} 4-\mathrm{H} 9$ and condition 5 of Theorem 1 are met.

2. There exists a positive solution $y$ of the inequality

$$
\delta\left(L_{n} x\right)(t)+F(t,(\mathcal{A} x)(t)) \leq 0
$$

such that $\lim _{t \rightarrow \infty}\left(L_{0} y\right)(t)>0$.

Then there exists a positive solution $x$ of equation (7) with the following properties:

$$
\begin{gathered}
\lim _{t \rightarrow \infty}\left(L_{0}\right)(t)>0 \\
0<\left(L_{i} x\right)(t) \leq\left(L_{i} y\right)(t) \text { eventually, } 0 \leq i \leq \ell-1, \ell \geq 1
\end{gathered}
$$

$0 \leq(-1)^{\ell+i}\left(L_{i} x\right)(t) \leq(-1)^{\ell+i}\left(L_{i} y\right)(t)$ eventually, $\ell \leq i \leq n-1, \quad \ell \leq n-1$, where $\ell$ is an integer, $0 \leq \ell \leq n$, such that $n+\ell$ is odd for $\delta=1$ and $n+\ell$ is even for $\bar{\delta}=-1$.

Theorem 8 is a corollary of Theorem 1 and Lemma 1.

Theorem 9: Let the following conditions hold:

1. Conditions $\mathrm{H} 1, \mathrm{H} 2, \mathrm{H} 4-\mathrm{H} 9$ and condition 4 of Theorem 2 are met.

2. $\quad$ There exists a negative solution $y$ of the inequality

$$
\delta\left(L_{n} x\right)(t)+F(t,(\mathcal{A} x)(t)) \geq 0
$$

such that $\lim _{t \rightarrow \infty}\left(L_{0} y\right)(t)<0$.

Then there exists a negative solution $x$ of equation (7) with the properties

$$
\begin{gathered}
\left(L_{i} y\right)(t) \leq\left(L_{i} x\right)(t) \text { eventually, } \ell>0,0 \leq i \leq \ell-1 \\
\lim _{t \rightarrow \infty}\left(L_{0} x\right)(t)<0
\end{gathered}
$$

$(-1)^{\ell+i}\left(L_{i} y\right)(t) \leq(-1)^{\ell+i}\left(L_{i} x\right)(t) \leq 0$ eventually, $\ell \leq n-1, \ell \leq i \leq n-1$ where $\ell$ is an integer $(0 \leq \ell \leq n)$ such that $n+\ell$ is odd for $\delta=1$ and $n+\ell$ is even for $\delta=-1$.

Theorem 9 is a corollary of Theorem 2 and Lemma 1.

Theorem 10: Let the following condition hold:

1. Conditions 1, 2 and 3 of Theorem 1 are met.

2. There exists a positive solution $y$ of equation (1) such that $\lim _{t \rightarrow \infty} \inf \left(L_{0} y\right)(t)>0$.

Then there exists a positive solution $x$ of equation (7) with the following properties:

1. $\quad \lim _{t \rightarrow \infty}\left(L_{0} x\right)(t)>0$

2. $x(t) \leq y(t)$ eventually.

Proof: Let $y$ be a positive solution of equation (1) in $\left[T_{0}, \infty\right), T_{0} \geq t_{0}$ such that $\lim _{t \rightarrow \infty}$ $\inf \left(L_{0} y\right)(t)>0$.

Introduce the following notation:

$$
\begin{gathered}
w_{0}(t)=w(t)-\frac{1}{\tau_{0}(t)} \lim _{t \rightarrow \infty} i n f\left(L_{0} y\right)(t) \\
v(t)=y(t)-w_{0}(t) .
\end{gathered}
$$


Then $\lim _{t \rightarrow \infty}\left(L_{0} v\right)(t)=\lim _{t \rightarrow \infty} \inf \left(L_{0} y\right)(t)>0$. From the fact that $\lim _{t \rightarrow \infty}\left(L_{0} v\right)(t)>0$ it follows that we can choose a constant $C$ such that $0<C<\lim _{t \rightarrow \infty}\left(L_{0} v\right)(t)$. Choose $T \geq T_{0}$ so that for $t \geq T$ the following inequalities are valid

$$
\left(L_{0} v\right)(t)>C>0 \text { and }\left(L_{0} w\right)(t) \geq-C .
$$

If we denote $\bar{v}(v)=v(t)-\frac{C}{\tau_{0}(t)}$ for $t \geq T$, then we obtain that

$$
0<\bar{v}(t) \leq y(t), \quad t \geq T .
$$

Then $\delta\left(L_{n} \bar{v}\right)(t)+F(t,(\mathcal{A} \bar{v})(t)) \leq 0$. Since $\lim _{t \rightarrow \infty}\left(L_{0} \bar{v}\right)(t)=\lim _{t \rightarrow \infty}\left(L_{0} v\right)(t)-C>0$, then from Theorem 8 , it follows that there exists a positive solution $x$ of equation (7) for which $\lim _{t \rightarrow \infty}\left(L_{0} x\right)(t)>0$ and $x(t) \leq \bar{v}(t) \leq y(t)$.

Theorem 11: Let the following conditions hold:

1. Conditions 1, 2, 3 and 4 of Theorem 2 are valid.

2. $\quad$ There exists a negative solution y of equation (1) such that $\lim _{t \rightarrow \infty} \sup \left(L_{0} y\right)(t)<0$.

Then there exists a negative solution $x$ of equation (7) with the following properties:

1. $\lim _{t \rightarrow \infty}\left(L_{0} x\right)(t)<0$,

2. $x(t) \geq y(t)$ eventually.

The proof of Theorem 11 is carried out along the scheme of the proof of Theorem 10.

\section{Some Particular Realizations of the Operator}

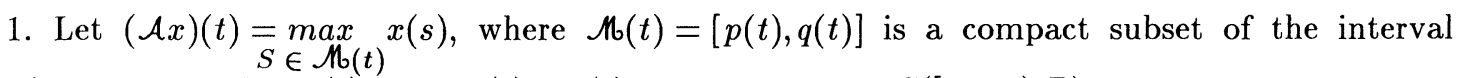
$\left[t_{0}, \infty\right), t \geq t_{0}$ and $\lim _{t \rightarrow \infty} p(t)=\infty, p(t) \leq q(t)$ for $t \geq t_{0}, p, q \in C\left(\left[t_{0}, \infty\right) ; \mathbb{R}\right)$.

We shall prove that for the so defined operator condition $H 5-H 8$ are satisfied.

In fact, if $0<x(t) \leq y(t)$ for $t \geq t_{0}$, then it is immediately verified that $0<(\mathcal{A} x)(t)=$ $\max _{S \in \mathcal{N}_{(}(t)} x(s)=\max _{S \in \mathcal{M}_{(t)}} y(s)=(\mathcal{A} y)(t)$ and $x(t)(\mathcal{A} x)(t)>0$ for $t \geq t_{0}$.

Let $x, x_{\kappa} \in C\left(\left[t_{0}, \infty\right) ; \mathbb{R}\right), \kappa=0,1, \ldots ; x_{\kappa}(t) \leq x(t)$ or $x_{\kappa}(t) \geq x(t)$ and $\lim _{\kappa \rightarrow \infty} x_{\kappa}(t)=x(t)$.

We shall prove that $\lim _{\kappa \rightarrow \infty}\left[\max _{S \in \mathcal{M}_{(}(t)} x_{\kappa}(x)\right]=\max _{S \in \mathcal{M}_{(}(t)} x(s)$.

To this end, we shall use the inequality

$$
\max _{S \in \mathcal{M}_{(}(t)} x(s)-\max _{S \in \mathcal{M}_{(t)}} y(s) \leq \max _{S \in \mathcal{M}(t)}[x(s)-y(s)] \quad(\mathrm{cf} .[9]) .
$$

From the fact that $x_{\kappa}(t) \underset{\kappa \rightarrow \infty}{\longrightarrow} x(t)$ for $t \geq t_{0}$ it follows that for each $\epsilon>0$ there exists $\kappa_{0}>0$ such that if $\kappa \geq \kappa_{0}$, then $\left|x_{\kappa}(t)-x(t)\right|<\epsilon$ for $t \geq t_{0}$.

Then

$$
\left|\max _{s \in \mathcal{M}(t)} x_{\kappa}(s)-\max _{s \in \mathcal{M}(t)} x(s)\right|
$$




$$
\begin{gathered}
=\left\{\begin{array}{lr}
\max _{s \in \mathcal{H}_{(}(t)} x_{\kappa}(s)-\max _{s \in \mathcal{H}(t)} x(s), & x_{\kappa}(t) \geq x(t) \\
\max _{s \in \mathcal{H}_{(}(t)} x(s)-\max _{s \in \mathcal{M}(t)} x_{\kappa}(s), & x_{\kappa}(t) \leq x(t)
\end{array}\right. \\
\leq\left\{\begin{array}{l}
\max _{s \in \mathcal{H}_{(}(t)}\left[x_{\kappa}(s)-x(s)\right]<\epsilon \\
\max _{s \in \mathcal{M}_{(}(t)}\left[x(s)-x_{\kappa}(s)\right]<\epsilon .
\end{array}\right.
\end{gathered}
$$

If $x \in \mathscr{D}_{n}$, then $\mathcal{A} x \in C\left(\left[T_{A x}, \infty\right) ; \mathbb{R}\right)($ cf. [1]).

Example 1: Consider the differential equation

$$
\left(t^{-1} x^{\prime}(t)\right)^{\prime}+\frac{1}{2} t^{-3} \max _{s \in[t-1, t]} x(s)=-t^{-2}, \quad t \geq 1
$$

and the differential inequality

$$
\left(t^{-1} x^{\prime}(t)\right)^{\prime}+\frac{1}{2} t^{-3} \max _{s \in[t-1, t]} x(s) \leq-t^{-2}, \quad t \geq 1
$$

Here $(\mathcal{A} x)(t)=\max _{s \in[t-1, t]} x(s)$. The functions $\tau_{0}(t)=1, \tau_{1}(t)=t^{-1}, F(t, u)=\frac{1}{2} u t^{-3}$ satisfy the conditions of Theorem 1 and $y(t)=4 t>0$ is a solution of (14). Then there exists a positive solution $x$ of equation (13) with the properties of (3).

For instance, $x(t)=2 t$ is such a solution.

Example 2: Consider the differential equation

$$
\left(t^{-1} x^{\prime}(t)\right)^{\prime}+\frac{1}{2} t^{-3} \max _{s \in[t, t+1]} x(s)=t^{-2}, \quad t \geq 1
$$

and the differential inequality

$$
\left(t^{-1} x^{\prime}(t)\right)^{\prime}+\frac{1}{2} t^{-3} \max _{s \in[t, t+1]} x(s) \geq t^{-2}, \quad t \geq 1 .
$$

Here $(\mathcal{A} x)(t)=\max _{s \in[t, t+1]} x(s)$. The functions $\tau_{0}(t)=1, \tau_{1}(t)=t^{-1}, F(t, u)=\frac{1}{2} t^{-3} u$ and $b(t)=$ $t^{-2}, w(t)=-t$ for $t \geq 1$ satisfy the conditions of Theorem 2 and $y(t)=-4 t<0$ is a solution of (16). Then there exists a negative solution $x$ of equation (15) with the properties (5). For instance, $x(t)=-2 t$ is such a solution.

Example 3: Consider the differential equation

$$
\left[e^{-t}\left[e^{-2 t}\left[e^{-t}\left[e^{t} x(t)\right]^{\prime}\right]^{\prime}\right]^{\prime}\right]^{\prime}-4 e^{-3 t} \max _{s \in[t-1, t]} x(s)=0, t \geq 1
$$

and the differential inequality

$$
\left[e^{-t}\left[e^{-2 t}\left[e^{-t}\left[e^{t} x(t)\right]^{\prime}\right]^{\prime}\right]^{\prime}\right]^{\prime}-4 e^{-3 t} \max _{s \in[t-1, t]} x(s) \geq 0, t \geq 1 .
$$

Here $(\mathcal{A} x)(t)=\max _{s \in[t-1, t]} x(s) . \quad$ The functions $\tau_{0}(t)=e^{t}, \quad \tau_{1}(t)=\tau_{3}(t)=e^{-t}, \quad \tau_{2}(t)=e^{-2 t}$, $F(t, u)=4 e^{-3 t} u$ and $\mathcal{H}(t)=[t-1, t]$ satisfy the conditions of Theorem 8 and $y(t)=e^{4 t}$ is a solution of (18) such that $\lim _{t \rightarrow \infty}\left(e^{t} \cdot e^{4 t}\right)=\infty$. Then there exists a positive solution $x$ of equation 
(17) with the properties (10). For instance, $x(t)=e^{t}$ is such a solution.

Example 4: Consider the differential equation

$$
\left[e^{-t}\left[e^{-2 t}\left[e^{-t}\left[e^{t} x(t)\right]^{\prime}\right]^{\prime}\right]^{\prime}\right]^{\prime}-4 e^{-3 t} \max _{s \in[t, t+1]} x(s)=0, t \geq 1
$$

and the differential inequality

$$
\left[e^{-t}\left[e^{-2 t}\left[e^{-t}\left[e^{t} x(t)\right]^{\prime}\right]^{\prime}\right]^{\prime}\right]^{\prime}-4 e^{-3 t} \max _{s \in[t, t+1]} x(s) \leq 0, t \geq 1 .
$$

Here $(\mathcal{A} x)(t)=\max _{s \in[t, t+1]} x(s)$. The functions $\tau_{0}(t)=e^{t}, \tau_{1}(t)=e^{-t}, \tau_{2}(t)=e^{-2 t}, \tau_{3}(t)=e^{-t}$, $F(t, u)=4 e^{-3} u$ satisfy the conditions of Theorem 9. Moreover, $y(t)=-e^{4 t}$ is a solution of inequality (20) such that $\lim _{t \rightarrow \infty}\left(L_{0} y\right)(t)<0$. Then there exists a negative solution $x$ of equation (19) with the properties (11). For instance, $x(t)=-e^{t}$ is a solution of equation (19) for which $\lim _{t \rightarrow \infty}\left(L_{0} x\right)(t)=-\infty$.

For $n=4$ and $\delta=-1$ we obtain that $\ell=2$. Then it is immediately verified that

$$
\begin{gathered}
\left(L_{i} y\right)(t) \leq\left(L_{i} x\right)(t)<0, \quad i=0,1 \\
(-1)^{2+i}\left(L_{i} y\right)(t) \leq(-1)^{2+i}\left(L_{i} x\right)(t)<0, \quad i=2,3 ; \quad t \geq 1 .
\end{gathered}
$$

Example 5: Consider the differential equations

$$
\begin{gathered}
{\left[t^{-1}\left[t^{-1} x^{\prime}(t)\right]^{\prime}\right]^{\prime}-3 t^{-5} \max _{s \in[t-1, t]} x(s)=-3 t^{-3}, t \geq 2} \\
{\left[t^{-1}\left[t^{-1} x^{\prime}(t)\right]^{\prime}\right]^{\prime}-3 t^{-5} \max _{s \in[t-1, t]} x(s)=0, \quad t \geq 2 .}
\end{gathered}
$$

Here $(\mathcal{A} x)(t)=\max _{s \in[t-1, t]} x(s)$. The functions $\tau_{0}(t)=1, \tau_{1}(t)=\tau_{2}(t)=t^{-1}, F(t, u)=3 t^{-5} u$, $b(t)=3 t^{-3}, w(t)=\frac{3}{4}\left[t^{2} \ln t-\frac{t^{2}}{2}\right]$ satisfy the conditions of Theorem 10. Moreover, $y(t)=t^{2}>0$ is a solution of equation (21) such that $\inf _{t>2} y(t)=4>0$. Then there exists a solution $x$ of equation (22) with the properties (12). For instance, $x(t)=t$ is a solution of equation (22) for which $\lim _{t \rightarrow \infty}\left(L_{0} x\right)(t)=\infty$ and $x(t)=t \leq t^{2}=y(t)$ for $t \geq 2$.

2. Let $(\mathcal{A} x)(t)=x(g(t))$, where $g \in C\left(\left[t_{0}, \infty\right) ; \mathbb{R}\right), \lim _{t \rightarrow \infty} g(t)=\infty$.

It is immediately verified that for the operator considered conditions $H 5-H 8$ are met.

Example 6: Consider the differential equation

$$
\left[e^{-t}\left[e^{-t} x^{\prime}(t)\right]^{\prime}\right]^{\prime}+2 e^{-4 t} x(2 t)=2 e^{-2 t}, \quad t \geq 2
$$

and the differential inequality

$$
\left[e^{-t}\left[e^{-t} x^{\prime}(t)\right]^{\prime}\right]^{\prime}+2 e^{-4 t} x(2 t) \leq 2 e^{-2 t}, \quad t \geq 2 .
$$

Here $(\mathcal{A} x)(t)=x(2 t)$. The functions $\tau_{0}(t)=1, \tau_{1}(t)=\tau_{2}(t)=e^{-t}, w(t)=t, F(t, u)=2 e^{-4 t} u$ and $b(t)=2 e^{-2 t}$ satisfy the conditions of Theorem 1. Moreover, $y(t)=t e^{t}$ is a solution of inequality (24) such that $\lim _{t \rightarrow \infty} \inf y(t)>0$. Then there exists a positive solution $x$ of equation (23) with the properties (3). 
For instance, $x(t)=e^{t}$ is a solution of equation (23), for which $\lim _{t \rightarrow \infty} \inf \left(L_{0} x\right)(t)>0$ and $x(t)=e^{t} \leq t e^{t}=y(t)$ for $t \geq 2$.

Example 7: Consider the differential equation

$$
\left[e^{-t}\left[e^{-t} x^{\prime}(t)\right]^{\prime}\right]^{\prime}+2 e^{-4 t} x(2 t)=-2 e^{-2 t}, t \geq 2
$$

and the differential inequality

$$
\left[e^{-t}\left[e^{-t} x^{\prime}(t)\right]^{\prime}\right]^{\prime}+2 e^{-4 t} x(2 t) \geq-2 e^{-2 t}, \quad t \geq 2 .
$$

Here $(\mathcal{A} x)(t)=x(2 t)$. The functions $\tau_{0}(t)=1, \tau_{1}(t)=\tau_{2}(t)=e^{-t}, w(t)=-t F(t, u)=2 e^{-4 t} u$, $b(t)=-2 e^{-2 t}$ satisfy the conditions of Theorem 2. Moreover, $y(t)=-t e^{t}$ is a solution of inequality (26). Then there exists a negative solution $x$ of equation (25) with the properties (5).

For instance, $x(t)=-e^{t}$ is such a solution.

Example 8: Consider the differential equation

$$
\left[t^{-1}\left[t^{-2} x(t)\right]^{\prime}\right]^{\prime}+t^{-6} x\left(3 t^{2}\right)=6 t^{-4}, t \geq 1 .
$$

Here $(\mathcal{A} x)(t)=x\left(3 t^{2}\right)$. The functions $w(t)=2 t, b(t)=6 t^{-4}, F(t, u)=t^{-6} u>0$ for $u \in \mathbb{R}_{+}$, $\tau_{0}(t)=t^{-2}$, and $\tau_{1}(t)=t^{-1}$ satisfy the conditions of Theorem 3 . Then each positive solution of equation (27) enjoys the property (6). For instance, $x(t)=t$ is such a solution for which $\lim _{t \rightarrow \infty} \frac{x(t)}{t^{2}}=0$.

Example 9: Consider the differential equation

$$
\left[e^{-t}\left[e^{t} x(t)\right]^{\prime}\right]^{\prime}-2 e^{-t} x(2 t)=0, \quad t \geq 2
$$

and the differential inequality

$$
-\left[e^{-t}\left[e^{t} x(t)\right]^{\prime}\right]^{\prime}+2 e^{-t} x(2 t) \geq 0, \quad t \geq 2 .
$$

Here $(\mathcal{A} x)(t)=x(2 t)$. The functions $\tau_{0}(t)=e^{t}, \quad \tau_{1}(t)=e^{-t}, \quad F(t, u)=2 e^{-t} u$ satisfy the conditions of Theorem 9. Moreover, $y(t)=-e^{2 t}$ is a solution of inequality (29) such that $\lim _{t \rightarrow \infty}\left(L_{0} y\right)(t)=-\infty$. Then there exists a negative solution $x$ of equation (28) with the properties (11).

For instance, $x(t)=-e^{t}$ is such a solution.

Example 10: Consider the differential equations

$$
\begin{gathered}
{\left[t^{-2}\left[t^{-1} x^{\prime}(t)\right]^{\prime}\right]^{\prime}-4 t^{-7} x\left(t^{2}\right)=-4 t^{-3}, t \geq 1} \\
{\left[t^{-2}\left[t^{-1} x^{\prime}(t)\right]^{\prime}\right]^{\prime}-4 t^{-7} x\left(t^{2}\right)=0, t \geq 1 .}
\end{gathered}
$$

Here $(\mathcal{A} x)(t)=x\left(t^{2}\right)$. The functions $\tau_{1}(t)=t^{-1}, \tau_{2}(t)=t^{-2}, F(t, u)=4 t^{-7} u, b(t)=-4 t^{-3}$, and $w(t)=\frac{2}{3} t^{3}$ satisfy the conditions of Theorem 10. Moreover, $y(t)=t^{2}$ is a solution of equation (30) such that $\lim _{t \rightarrow \infty}$ inf $y(t)>0$. Then there exists a positive solution $x$ of equation (31) with the properties (12). For instance, $x(t)=t>0$ is a solution of equation (31) for which $\lim _{t \rightarrow \infty}\left(L_{0} x\right)(t)=\infty, x(t)=t \leq t^{2}=y(t), t \geq 1$. 
3. Let $(\mathcal{A} x)(t)=\int_{t}^{t} \kappa(t, s) x(s) d s$ where $a$ is a positive constant, $\kappa \in C\left(\left[t_{0}+a, \infty\right)^{2} ;(0, \infty)\right)$ and there exists a constant $c>0$ such that $\kappa(t, u) \leq c$ eventually.

We shall prove that for the operator consider conditions $\mathrm{H}$ 5- $\mathrm{H} 8$ are met.

If $0<x(t) \leq y(t)$, then

$$
(\mathcal{A} y)(t)-(\mathcal{A} x)(t)=\int_{t-a}^{t} \kappa(t, x)[y(s)-x(s)] d s \geq 0 .
$$

It is immediately verified that conditions $H 5$ and $H 9$ hold. Let

$$
x_{\kappa}, x \in \mathfrak{D}_{n}, \quad \kappa=0,1, \ldots, \lim _{\kappa \rightarrow \infty} x_{\kappa}(t)=x(t), \text { i.e. for any } \epsilon>0
$$

and each fixed number $t \geq t_{0}$ there exists $\kappa_{0}>0$ such that for $\kappa \geq \kappa_{0}$ we have $\mid x_{\kappa}(t)-$ $x(t) \mid<\frac{\epsilon}{c a}$.

Then

$$
\lim _{\kappa \rightarrow \infty}\left(\mathcal{A} x_{\kappa}\right)(t)=(\mathcal{A} x)(t)
$$

Example 11: Consider the differential equation

$$
\left[e^{-t}\left[e^{-t} x^{\prime}(t)\right]^{\prime}\right]^{\prime}+\int_{t-1}^{t} e^{s-t} x(s) d s=\frac{e^{2}-1}{2 e^{2}} e^{t}, t \geq 1
$$

and the differential inequality

$$
\left[e^{-t}\left[e^{-t} x^{\prime}(t)\right]^{\prime}\right]^{\prime}+\int_{t-1}^{t} e^{s-t} x(s) d s \leq \frac{e^{2}-1}{2 e^{2}} e^{t}, t \geq 1 .
$$

Here $(\mathcal{A} x) t)=\int_{t}^{t} e^{s-t} x(s) d s . \quad$ The functions $\quad \tau_{0}(t)=1, \quad \tau_{1}(t)=\tau_{2}(t)=e^{-t}, \quad F(t, u)=u$, $\kappa(t, s)=e^{s-t}$, and $w(t)=\frac{e^{2}-1}{12 e^{2}} e^{3 t}$ satisfy the conditions of Theorem 1. Moreover, $y(t)=t e^{t}$ is a solution of inequality (33) such that $\lim _{t \rightarrow \infty} \inf \left(L_{0} y\right)(t)>0$. Then there exists a positive solution $x$ of equation (32) with the properties (3).

For instance, $x(t)=e^{t}$ is such a solution.

Example 12: Consider the differential equation

and the differential inequality

$$
\left[e^{-t}\left[e^{-t} x^{\prime}(t)\right]^{\prime}\right]^{\prime}+\int_{t-1}^{t} e^{s-t} x(s) d s=\frac{1-e^{2}}{2 e^{2}} e^{t}, \quad t \geq 1
$$

$$
\left[e^{-t}\left[e^{-t} x^{\prime}(t)\right]^{\prime}\right]^{\prime}+\int_{t-1}^{t} e^{s-t} x(s) d s \geq \frac{1-e^{2}}{2 e^{2}} e^{t}, \quad t \geq 1 .
$$

Here $(\mathcal{A} x)(t)=\int_{t-1}^{t} e^{s-t} x(s) d s . \quad$ The functions $\tau_{0}(t)=1, \quad \tau_{1}(t)=\tau_{2}(t)=e^{-t}, \quad F(t, u)=u$, $\kappa(t, s)=e^{s-t} \leq 1, \quad b(t)=\frac{1-e^{2}}{2 e^{2}} e^{t}$, and $w(t)=\frac{1-e^{2}}{12 e^{2}}, e^{3 t}$ satisfy the conditions of Theorem 2. 
Moreover, $y(t)=-t e^{t}$ is a solution of inequality (35).

Then there exists a negative solution $x$ of equation (34) with the properties (5).

For instance, $x(t)=-e^{t}$ is such a solution.

\section{Acknowledgement}

The present investigation is supported by the Bulgarian Ministry of Science and Education under Grant MM-7.

\section{References}

[1] Angelov, V.G., Bainov, D.D., On the functional differential equations with "maximums", Applicable Analysis 16 (1983), 187-194.

[2] Atkinson, F.V., On second-order differential inequalities, Proc. Roy. Soc. Edinburgh, Sect. A 72 (1972-73), 109-127.

[3] Kartsatos, A.G., On $n$-th order differential inequalities, J. Math. Anal. Appl. 52 (1975), 1-9.

[4] Kartsatos, A.G., Onose, H., A comparison theorem for functional differential equations, Bull. Austral. Math. Soc. 14 (1976), 343-347.

[5] Magomedov, A.R., On some problems of differential equations with "maxima", Izv. Acad. Sci. Azerb. SSR, Ser. Phys.-Techn. and Math. Sci. 108:1 (1977), 104-108 (in Russian).

[6] Onose, H., A comparison theorem and the forced oscillation, Bull. Austral. Math. Soc. 13 (1975), 13-19.

[7] Onose, H., A comparison theorem for delay differential equations, Utilitas Math. 10 (1976), 185-191.

[8] Popov, E.P., Automatic Regulation and Control, Nauka, Moscow 1966 (in Russian).

[9] Simeonov, P.S., Bainov, D.D., Application of the method of bilateral approximations to the investigation of periodic systems of ordinary differential equations with maxima, Archivum Mathematicum (Brno) 21:2 (1985), 65-76.

[10] Staikos, V.A., Asymptotic behavior and oscillation of bounded solutions of differential equations with deviating arguments, Ukr. Math. J. 31 (1979), 705-716 (in Russian).

[11] Staikos, V.A., Philos, Ch. G., Basic comparison results for the oscillatory and asymptotic behavior of differential equations with deviating arguments, Bull. of Inst. Math. Academia Sinica 9 (1981), 417-445. 


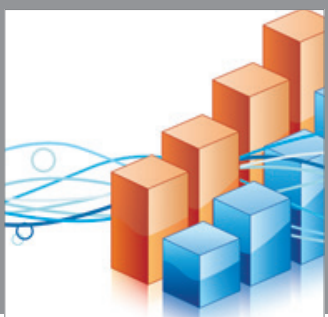

Advances in

Operations Research

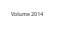

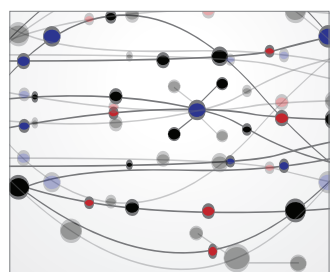

\section{The Scientific} World Journal
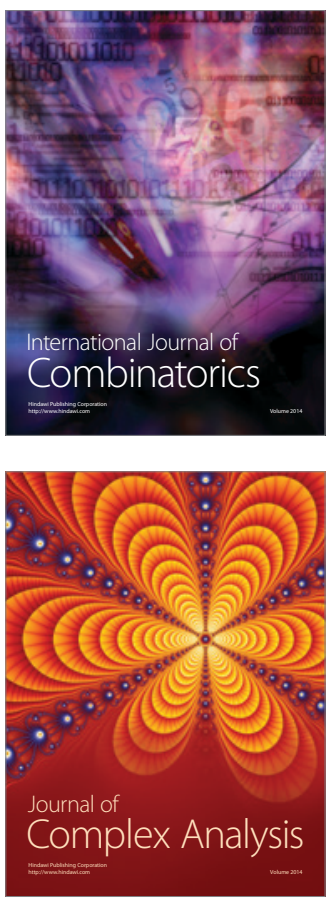

International Journal of

Mathematics and

Mathematical

Sciences
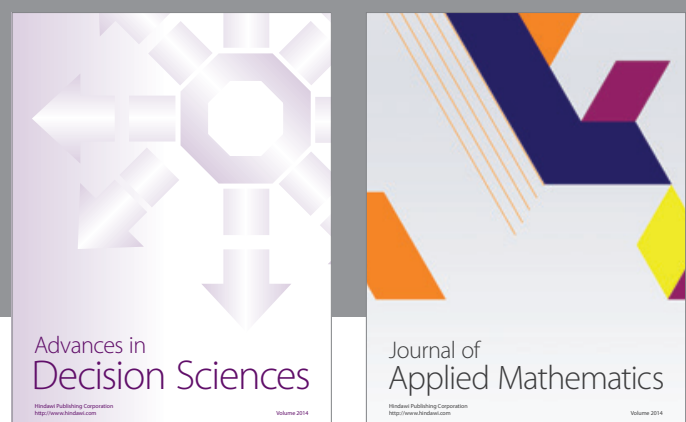

Journal of

Applied Mathematics
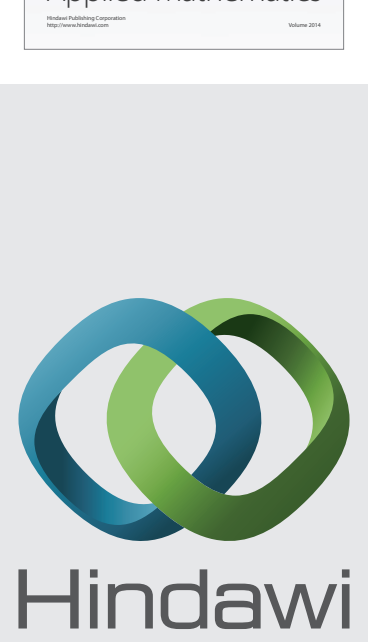

Submit your manuscripts at http://www.hindawi.com
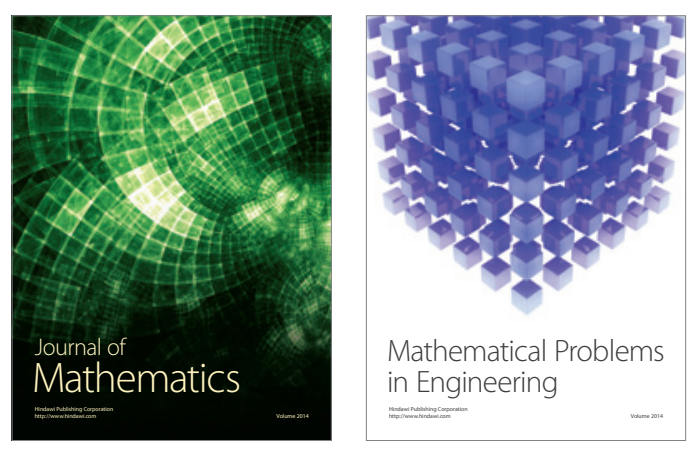

Mathematical Problems in Engineering
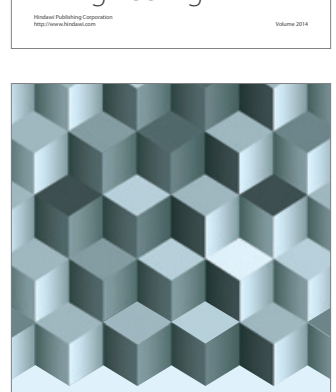

Journal of

Function Spaces
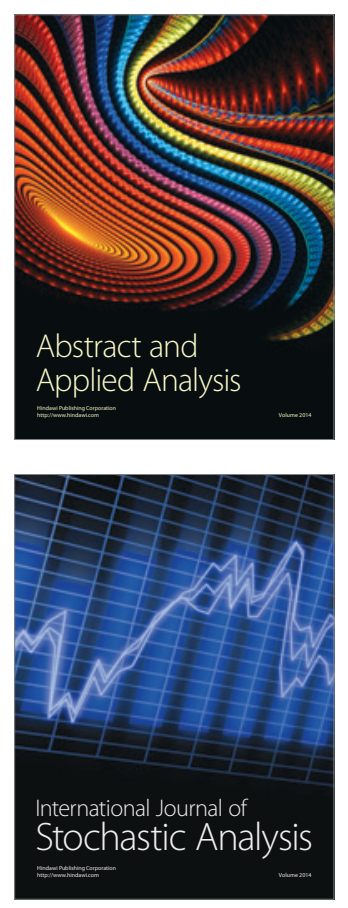

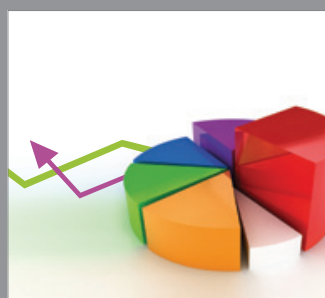

ournal of

Probability and Statistics

Promensencen
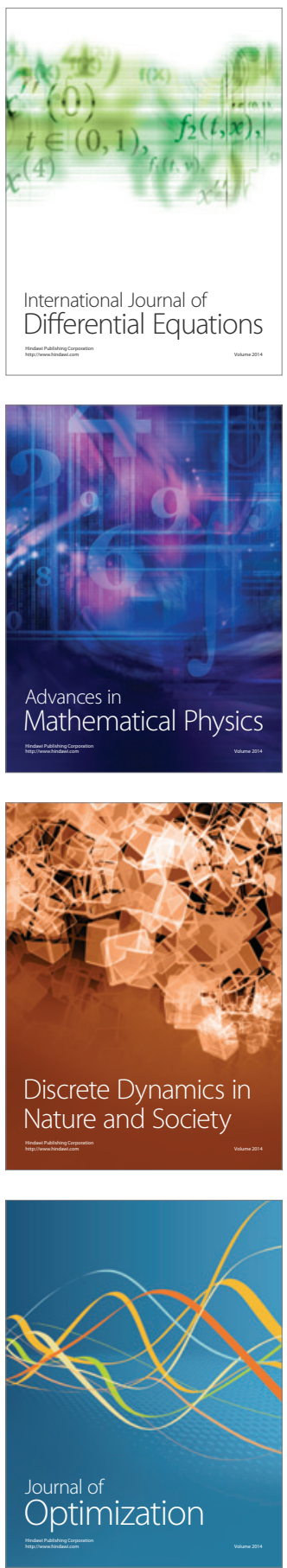